\title{
Ginkgo leaf sign: a highly predictive imaging feature of spinal meningioma
}

\author{
Satoshi Yamaguchi, MD, PhD, ${ }^{1}$ Masaaki Takeda, MD, PhD, ${ }^{1}$ Toshiyuki Takahashi, MD, PhD, ${ }^{2}$ \\ Hitoshi Yamahata, MD, PhD, ${ }^{3}$ Takafumi Mitsuhara, MD, PhD, ${ }^{1}$ Tadaaki Niiro, MD, PhD, ${ }^{3}$ \\ Junya Hanakita, MD, PhD, ${ }^{2}$ Kazutoshi Hida, MD, PhD, ${ }^{4}$ Kazunori Arita, MD, PhD, ${ }^{3}$ and \\ Kaoru Kurisu, MD, PhD'
}

\begin{abstract}
1Department of Neurosurgery, Hiroshima University, Graduate School of Biomedical and Health Sciences, Hiroshima; ${ }^{2}$ Fujieda Heisei Memorial Hospital, Fujieda, Shizuoka; ${ }^{2}$ Department of Neurosurgery, Kagoshima University, Graduate School of Medical and Dental Sciences, Kagoshima; and ${ }^{4}$ Sapporo Azabu Neurosurgical Hospital, Sapporo, Hokkaido, Japan
\end{abstract}

\begin{abstract}
OBJECT Spinal meningioma and schwannoma are the most common spinal intradural extramedullary tumors, and the differentiation of these 2 tumors by CT and MRI has been a matter of debate. The purpose of this article is to present a case series of spinal meningiomas showing unique imaging features: a combination of a fan-shaped spinal cord and a streak in the tumor. The authors termed the former imaging feature "ginkgo leaf sign" and evaluated its diagnostic value. METHODS The authors present 7 cases of spinal meningioma having the ginkgo leaf sign. Thirty spinal extramedullary tumors arising lateral or ventrolateral to the spinal cord were studied to evaluate the diagnostic value of the ginkgo leaf sign for spinal meningiomas. Among 30 cases, 12 tumors were spinal meningiomas and 18 tumors from the control group were all schwannomas.
\end{abstract}

RESULTS Seven of the 12 spinal meningiomas were positive for the ginkgo leaf sign. The sign was not present in the control group tumors. The overall ability to use the ginkgo leaf sign to detect meningioma indicated a sensitivity of $58 \%$, specificity of $100 \%$, positive predictive value of $100 \%$, and negative predictive value of $78 \%$.

CONCLUSIONS The ginkgo leaf sign is highly specific to spinal meningiomas arising lateral or ventrolateral to the spinal cord. In the present series, the ginkgo leaf sign was perfectly predictive for spinal meningioma.

http://thejns.org/doi/abs/10.3171/2015.3.SPINE1598

KEY WORDS spinal meningioma; magnetic resonance imaging; dentate ligament; oncology

$\mathrm{I}$ NTRADURAL extramedullary tumors account for $80 \%$ of all adult intraspinal tumors. Among them, meningiomas and schwannomas are the most common, and they comprise $55 \%$ of all spinal extramedullary tumors. ${ }^{1}$ Clinicians have tried to distinguish meningiomas and schwannomas on MR images by using the following characteristics: meningiomas are usually identified as having iso- to slightly hyposignal intensity on T1-weighted MR images (T1WIs) and iso- to slightly hypersignal intensity on T2-weighted MR images (T2WIs). The tumor is homogeneously enhanced with gadolinium-diethylenetriamine pentaacetic acid (Gd-DTPA) and often accompanies the dural tail sign. ${ }^{6,11}$ Schwannomas generally show iso- to hyposignal intensity on T1WIs, and hyper- or mixed-signal intensity on T2WIs. The tumor is well enhanced with Gd-DTPA but often shows ring-like or irregular enhancement, depending on the degenerative changes within the tumor. ${ }^{3,6,11}$ However, in the clinical settings, differentiation of these tumors from signal intensity alone is often difficult. ${ }^{6,8,9}$ Location and configuration of the tumor may be another hint; meningiomas often arise posterolaterally in the thoracic spine and anteriorly in the cervical spine, whereas schwannomas generally originate from the dorsal rootlets and are usually found in the dorsal spinal cord or

ABBREVIATIONS $\mathrm{CP}=$ cerebellopontine; Gd-DTPA = gadolinium-diethylenetriamine pentaacetic acid; T1WI = T1-weighted MR image; T2WI = T2-weighted MR image SUBMITTED January 26, 2015. ACCEPTED March 26, 2015

INCLUDE WHEN CITING Published online July 31, 2015; DOI: 10.3171/2015.3.SPINE1598.

DISCLOSURE The authors report no conflict of interest concerning the materials or methods used in this study or the findings specified in this paper. 
dorsolateral to the spinal cord. The tumor may protrude through the neural foramen, forming a dumbbell-shaped mass. ${ }^{1,11}$ However, we sometimes encounter exceptional cases such as dumbbell-shaped meningiomas and ventral schwannomas. ${ }^{1,12}$ Thus, differentiation of spinal meningiomas and schwannomas on neuroimaging is not always reliable and remains a matter of debate. ${ }^{3,6-8}$ In this report, we describe a case series of spinal meningiomas demonstrating unique imaging features. Also, we propose the "ginkgo leaf sign" as highly specific to spinal meningiomas.

\section{Methods}

Between 2004 and 2014, we surgically treated a total of 30 patients with spinal intradural extramedullary tumors arising lateral or ventrolateral to the spinal cord. This retrospective study to examine these cases was approved by the institutional review board of Hiroshima University. Data were collected from medical charts and radiological review, and all data were deidentified. Of the 30 cases of spinal intradural extramedullary tumors, 12 cases were spinal cord meningiomas and 18 cases were schwannomas. Among the 12 cases of meningioma, we found 7 cases in which contrast-enhanced axial MR images showed a fan-shaped spinal cord and a streak in the tumor. We named this MRI finding the ginkgo leaf sign because the shape of the deformed cord and the streak on MR images resembled a ginkgo leaf. To confirm this sign as specific to meningiomas, MR images of meningiomas and other extramedullary tumors that arose lateral or ventrolateral to the spinal cord were retrospectively reviewed. The study describes 7 cases of surgically treated spinal meningiomas that demonstrate the unique MRI feature, the ginkgo leaf sign.

\section{Comparative Study to Evaluate the Usefulness of the Ginkgo Leaf Sign}

We retrospectively evaluated the positivity of the ginkgo leaf sign in intraspinal extramedullary nonmenigioma tumors of the control group. All tumors of the control group treated during the research period were schwannomas. We evaluated the sensitivity, specificity, and positive and negative predictive values of the ginkgo leaf sign for meningioma. Of the 30 cases surgically treated between 2004 and 2014, 12 cases (including the aforementioned 7 cases) were meningiomas (Cases 1-12: meningioma group) and 18 cases were schwannomas (Cases 13-30: control group). We excluded the tumors below the L-2 level because the spinal cord and dentate ligament are absent below this level. The authors (S.Y. and M.T.) reevaluated the preoperative sagittal and axial contrast-enhanced MR images to confirm if the ginkgo leaf sign was present.

\section{Illustrative Cases}

\section{Case 1. Spinal Meningioma Demonstrating the Ginkgo Leaf Sign}

A 74-year-old female presented with cervical myelopathy. Sagittal T1WIs with Gd-DTPA showed an extramedullary mass compressing the spinal cord at the C5-6 level
(Fig. 1 left). Axial images showed that the tumor occupied the right half of the spinal canal, displacing the spinal cord to the left side. The displaced spinal cord was deformed like a fan. A low-signal-intensity streak originating from the spinal cord was seen in the tumor. The combination of the fan-shaped cord and the streak resembled a ginkgo leaf (Fig. 1 right). The patient underwent total resection of the tumor. Intraoperative findings confirmed that the dentate ligament was involved with the tumor, and because of ligament involvement, the tumor was separated into ventral and dorsal compartments. The tumor appeared to originally arise from the ventrolateral surface of the dura, as feeding arteries were most abundant in this area (Fig. 2A-D). Surgical findings revealed that a low-signal-intensity streak on the MR image corresponded to the involved dentate ligament in the tumor. The histopathological diagnosis was meningioma.

\section{Case Series of Meningiomas Positive for the Ginkgo Leaf Sign}

MR images obtained in all patients with meningiomas positive for the ginkgo leaf sign, except for Case 1, are shown in Fig. 3. With regard to the spinal level, the tumors were located from the C5-6 level to the T12-L1 level: C5-6 level in Cases 2 and 3; T-2 level in Case 4; T12-L1 level in Case 5; T8-9 level in Case 6; and T-12 level in Case 7. Cervical and upper thoracic tumors (Cases 1-4) showed a low-signal-intensity streak more clearly than middle to lower thoracic tumors (Cases 5-7).

\section{Results}

The results of comparative study of the positive and negative cases of ginkgo leaf sign in both groups are shown in Table 1. As was aforementioned, 7 of 12 meningiomas were positive for ginkgo leaf sign. The sign was negative in all control group tumors. Overall, the ginkgo leaf sign was able to detect meningioma with a sensitivity of $58 \%$, specificity of $100 \%$, positive predictive value of $100 \%$, and negative predictive value of $78 \%$. We conclude that the ginkgo leaf sign is highly specific to spinal meningiomas arising lateral or ventrolateral to the spinal cord. In the present series, the presence of the ginkgo leaf sign was perfectly predictive for spinal meningioma.

\section{Discussion}

\section{Differentiation of Spinal Meningiomas and Schwannomas on Neuroimaging}

Every intradural extramedullary tumor should be considered a possible meningioma or schwannoma because they are the most common intradural extramedullary neoplasms in this region., ${ }^{1,2}$ However, differentiating these 2 tumors has been difficult, especially in earlier MRI studies, probably due to lower signal-to-noise ratio and poor spatial resolution. ${ }^{8}$ Recent studies have focused on trying to differentiate these tumors, looking especially at signal intensity on T2WIs and enhancement pattern on Gd-enhanced MR images. Liu et al. retrospectively studied the effectiveness of CT and MRI to differentiate spinal meningiomas and schwannomas in 128 patients. The result 


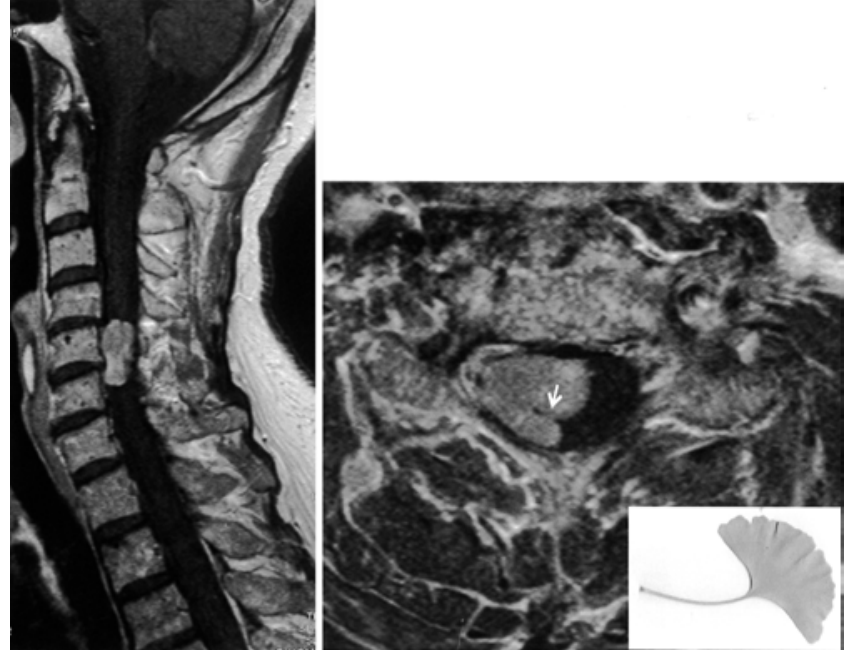

FIG. 1. Case 1. MR images obtained in a 74-year-old female with cervical myelopathy, a representative case of spinal meningioma with ginkgo leaf sign. Left: Sagittal T1WI with Gd-DTPA showing an extramedullary mass compressing the spinal cord at the C5-6 level. Right: Axial T1Wl with Gd-DTPA showing the tumor displacing the spinal cord to the contralateral side, yielding a fan-shaped deformity and a low-signal-intensity streak (arrow). Inset: Photograph showing a sample of ginkgo leaf: leaf blade corresponds to displaced spinal cord, and a leaf stalk corresponds to a low-signal-intensity streak on MR image.

showed that positive predictors of meningiomas were the dural tail sign on contrast-enhanced T1WIs and calcification on CT scans. For schwannomas, fluid signal intensity of the tumor on T2WIs, rim enhancement on T1WIs with Gd-DTPA, and scalloping on CT scans were predictive factors. ${ }^{6}$ De Verdelhan et al. retrospectively reviewed MR images obtained in 52 patients with spinal schwannomas and meningiomas. The authors concluded that a diagnosis of schwannoma should be made when the tumor showed hypersignal intensity on T2WIs or intense contrast enhancement without dural tail signs. ${ }^{3}$ Setzer et al. mentioned hints for differentiating meningiomas and schwannomas: dural tail and broad dural contrast-enhancement are predictive for meningioma, and more lateral location suggests schwannomas. However, they concluded that a reliable differentiation of these 2 entities is not possible with MRI or other imaging techniques. ${ }^{9}$

\section{Ginkgo Leaf Sign is Highly Specific to Spinal Meningiomas}

The leaf of the ginkgo tree, Ginkgo biloba, has a fanshaped blade and a slightly curved leafstalk (Fig. 1 inset). In this retrospective study, we presented unique findings on contrast-enhanced MR images common to all 7 cases of spinal meningiomas: fan-shaped deformity of the spinal cord and a streak in the tumor. Since these features looked like a ginkgo leaf, we coined the term "ginkgo leaf sign," which we have shown to be highly predictive of spinal meningiomas. Surgical findings revealed that the ginkgo leaf shape visible in MR images was deformed spinal cord, and what appears as a streak was a stretched dentate ligament involved with the tumor. All 7 tumors had dural attachment to the ventrolateral side of the spinal cord. These findings suggest that the tumor originated on the ventral side of the dentate ligament. Although the fibers of the dentate ligament have longitudinally broad origin on the lateral surface of the spinal cord, the fibers converge at the site of ligament attachment to the dura, creating an open
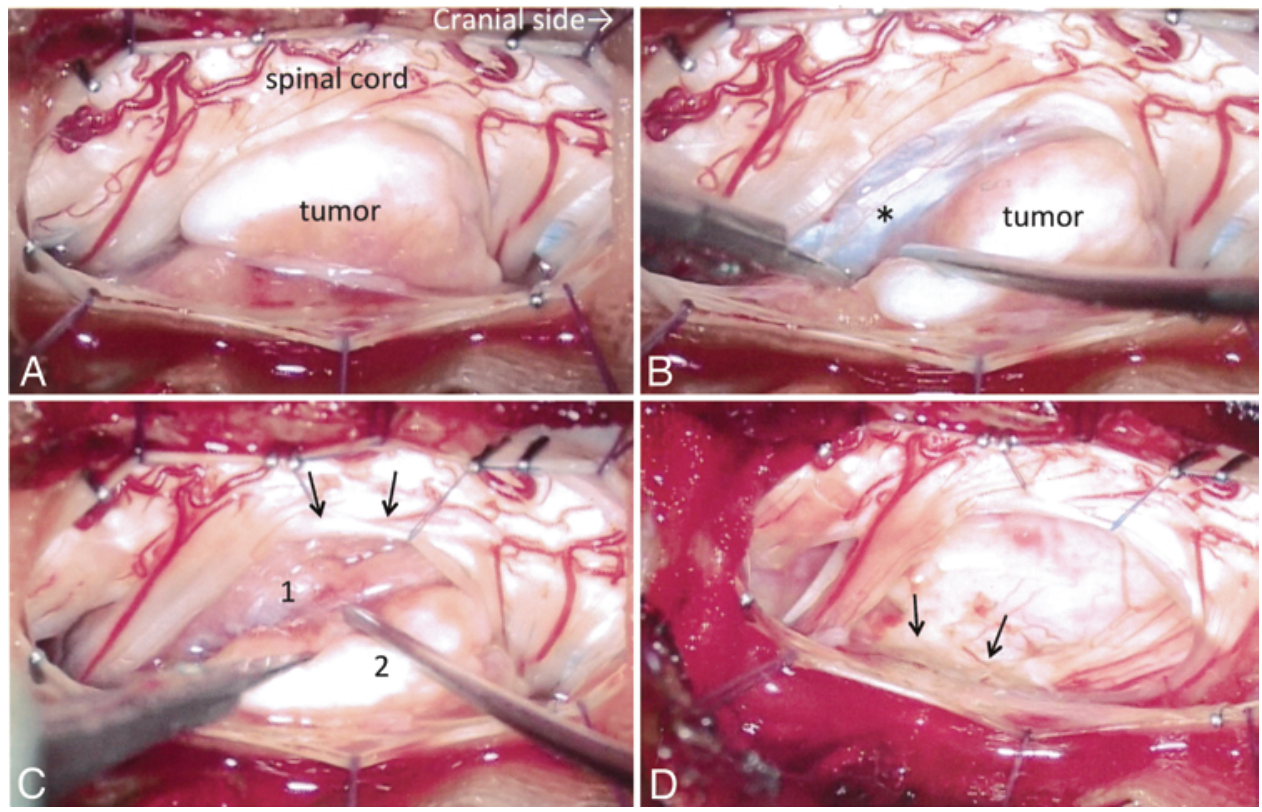

FIG. 2. Case 1. Serial intraoperative photographs showing meningioma at the C5-6 level. A: The tumor is markedly displacing the spinal cord to the contralateral side. B: Retraction of the dorsal part of the tumor exposing the dentate ligament $\left({ }^{*}\right)$ involved in the tumor. C: View after reflection of the dentate ligament toward the spinal cord (arrows). The tumor is separated into ventral (1) and dorsal (2) compartments by the ligament. D: View after total removal of the tumor. The feeding arteries are most abundant in the ventrolateral side of the dura (arrows). This finding implies the tumor arose originally in this area. 

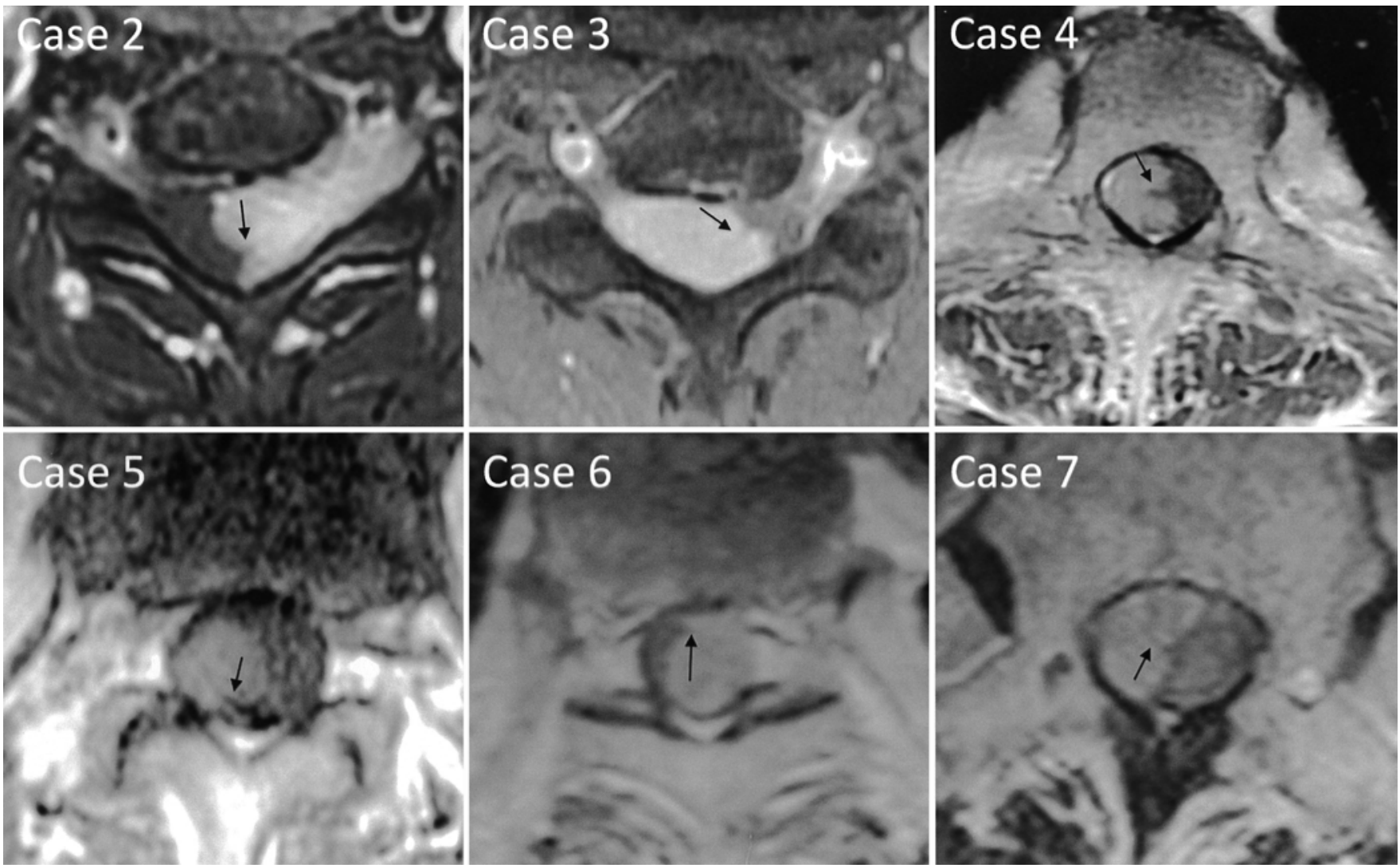

FIG. 3. Axial contrast-enhanced T1-weighted MR images obtained in Cases 2-7 showing the presence of the ginkgo leaf sign in spinal meningiomas. An arrow in each image indicates a low-signal-intensity streak corresponding to the involved dentate ligament in the tumor. Cervical and upper thoracic tumors (Cases 2-4) show the streak more clearly than middle to lower thoracic tumors (Cases 5-7). The tumors are compressing the spinal cord at the C5-6 level (Cases 2 and 3), T-2 level (Case 4), T12-L1 level (Case 5), T8-9 level (Case 6), and T-12 level (Case 7).

space between the ligament and dura. ${ }^{10}$ We estimate that the ventral tumor extended to the dorsal side through this space. An enlarged tumor will deform the spinal cord like a fan because the lateral side of the spinal cord is tethered to the dura by the stretched dentate ligament. The present study demonstrates that all control-group tumors, being schwannomas, did not demonstrate the ginkgo leaf sign; thus, the study concluded that this sign was highly specific to meningioma. Why the ginkgo leaf sign was not visible in images of ventral or ventrolateral schwannomas is probably due to a difference in growth pattern of these tumors. The cerebellopontine (CP) angle is an anatomical space where both meningioma and vestibular schwannoma

TABLE 1. Overall ability of ginkgo leaf sign to predict spinal meningioma

\begin{tabular}{lcccc}
\hline $\begin{array}{c}\text { Ginkgo } \\
\text { Leaf Sign }\end{array}$ & $\begin{array}{c}\text { Meningioma Group } \\
(n=12)\end{array}$ & $\begin{array}{c}\text { Control Group } \\
(n=18)\end{array}$ & PPV & NPV \\
\hline Positive & 7 & 0 & $100 \%$ & \\
\hline Negative & 5 & 18 & & $78 \%$ \\
\hline Sensitivity & $58 \%$ & & & \\
\hline Specificity & & $100 \%$ & & \\
\hline
\end{tabular}

$\mathrm{NPV}=$ negative predictive value; $\mathrm{PPV}=$ positive predictive value. are likely to arise. In the $\mathrm{CP}$ angle, enlarging vestibular schwannomas displace surrounding cranial nerves such as the facial nerve and trigeminal nerve. Surgical findings usually demonstrate that displaced cranial nerves are not involved in the tumor, but these nerves are stretched and adhere to the tumor surface. ${ }^{5}$ On the contrary, meningiomas often encircle surrounding nerves and vessels, involving them within the tumor body. ${ }^{4} \mathrm{As}$ seen in the $\mathrm{CP}$ angle, enlarged spinal schwannomas arising on the ventral side seem to simply stretch the dentate ligament over the surface of the tumor. This growth pattern will result in the absence of ginkgo leaf sign.

\section{Study Limitations}

All tumors in the control group were schwannomas. Because of the lack of other extramedullary tumors such as neurofibromas and paragangliomas in the present study, we cannot definitively conclude that the ginkgo leaf sign is predictive exclusively for meningiomas.

\section{Conclusions}

Within our limited patient groups, the unique imaging feature of the ginkgo leaf sign was highly specific to spinal meningiomas arising lateral or ventrolateral to the spi- 
nal cord. Although further validation of this sign is warranted in larger case studies, its high predictive ability will be useful in clinical imaging diagnosis.

\section{References}

1. Abul-Kasim K, Thurnher MM, McKeever P, Sundgren PC: Intradural spinal tumors: current classification and MRI features. Neuroradiology 50:301-314, 2008

2. Chamberlain MC, Tredway TL: Adult primary intradural spinal cord tumors: a review. Curr Neurol Neurosci Rep 11:320-328, 2011

3. De Verdelhan O, Haegelen C, Carsin-Nicol B, Riffaud L, Amlashi SF, Brassier G, et al: MR imaging features of spinal schwannomas and meningiomas. J Neuroradiol 32:42-49, 2005

4. Hu YF, Cheng PW, Young YH: Comparison of vestibular function between large cerebellopontine angle meningioma and schwannoma. Acta Otolaryngol 129:161-165, 2009

5. Kulwin CG, Cohen-Gadol AA: Technical nuances of resection of giant $(>5 \mathrm{~cm})$ vestibular schwannomas: pearls for success. Neurosurg Focus 33(3):E15, 2012

6. Liu WC, Choi G, Lee SH, Han H, Lee JY, Jeon YH, et al: Radiological findings of spinal schwannomas and meningiomas: focus on discrimination of two disease entities. Eur Radiol 19:2707-2715, 2009

7. Matsumoto S, Hasuo K, Uchino A, Mizushima A, Furukawa $\mathrm{T}$, Matsuura Y, et al: MRI of intradural-extramedullary spinal neurinomas and meningiomas. Clin Imaging 17:46-52, 1993

8. Schroth G, Thron A, Guhl L, Voigt K, Niendorf HP, Garces LR: Magnetic resonance imaging of spinal meningiomas and neurinomas. Improvement of imaging by paramagnetic contrast enhancement. J Neurosurg 66:695-700, 1987
9. Setzer M, Vatter H, Marquardt G, Seifert V, Vrionis FD: Management of spinal meningiomas: surgical results and a review of the literature. Neurosurg Focus 23(4):E14, 2007

10. Tubbs RS, Salter G, Grabb PA, Oakes WJ: The denticulate ligament: anatomy and functional significance. J Neurosurg 94 (2 Suppl):271-275, 2001

11. Wein S, Gaillard F: Intradural spinal tumours and their mimics: a review of radiographic features. Postgrad Med J 89:457-469, 2013

12. Yamahata H, Yamaguchi S, Mori M, Kubo F, Tokimura H, Arita K: Ventral schwannoma of the thoracolumbar spine. Asian Spine J 7:339-344, 2013

\section{Author Contributions}

Conception and design: Yamaguchi, Niiro, Hanakita, Hida, Arita, Kurisu. Acquisition of data: Yamaguchi, Takeda, Takahashi, Yamahata, Hanakita, Hida. Analysis and interpretation of data: Yamaguchi, Takeda. Drafting the article: Yamaguchi. Critically revising the article: Mitsuhara.

\section{Supplemental Information}

Previous Presentation

Portions of this work were presented in 2014, at the 29th Annual Meeting of the Japanese Society of Spinal Surgery (June 12-13, Tokyo, Japan).

\section{Correspondence}

Satoshi Yamaguchi, Department of Neurosurgery, Hiroshima University Graduate School of Biomedical and Health Sciences, 1-2-3 Kasumi, Minami-ku, Hiroshima-city, Hiroshima 734-8551, Japan.email: satoya@hiroshima-u.ac.jp. 